\title{
A Neural Prediction Model to Predict Power Load Involving Weather Element
}

\author{
XinLei Li \\ School of energy,power and mechanical engineering,North China Electric Power University,Baoding \\ 071000, China \\ lixinlei24@163.com
}

\begin{abstract}
This paper using the BP neural network model to predict the load of power system. This paper takes weather element into account, quantifying the weather element and making them as the input variable. According to the contrast to the predicted model without considering weather element, the results show that the former performs better.
\end{abstract}

Keywords: The load of power system, weather element, BP neural network

\section{Introduction}

Power load forecasting, especially the short-term load forecasting system, plays an important role in arranging power grid operation. A precise short-term load forecast is very important to the social's economy, security, and development. [1]

In the process of power load forecasting, artificial neural network has the merits of nonlinearity, self-organization, self-adaption, and massively parallel processing [2], which can fit any Arbitrary complex nonlinear relationship conveniently. At the same time, adding the weather element to the forecasting process can make the conclusion more accurately [3].

\section{Establishment of the model}

\subsection{Quantifying the weather element}

This paper utilizing the BP model to predict the load of power system. In order to consider the influence of the weather, this paper quantify the weather element to put it as the input variable of neural network model.

The temperature has a similarly effect to load when it varies within a certain scope. Thus, we periods process the temperature and quantify it according to the adaption of human to temperature Consequently, we quantify temperature of $-10^{\circ} \mathrm{C}$ to $40^{\circ} \mathrm{C}$ to $(-1,-0.8,-0.6,-0.4,0,0.4,0.6,0.8,1)$.

The precipitation plays an important role in electrical load. We can quantify the precipitation to the Tab. 1 owing to the amount of precipitation. And the humidity can be quantified as Tab. 2 .

Tab. 1 The quantization table of precipitation

\begin{tabular}{ccccccc}
\hline precipitatio & No rain & Little rain & Moderate & Heavy & Intense & downpour \\
$n$ & $/ 0$ & $/ 15$ & rain & rain & fall & /200 \\
/millimeter & millimete & millimeter & $/ 30$ & $/ 60$ & $/ 120$ & millimeter \\
& $r$ & & millimeter & millimeter & millimeter & \\
\hline $\begin{array}{c}\text { quantized } \\
\text { value }\end{array}$ & 0 & 0.2 & 0.4 & 0.6 & 0.8 & 1 \\
\hline
\end{tabular}

Tab. 2 The quantization table of humidity

\begin{tabular}{ccccc}
\hline humidity & $40 \%$ & $60 \%$ & $80 \%$ & $100 \%$ \\
\hline quantized value & 0 & 0.3 & 0.6 & 1 \\
\hline
\end{tabular}




\subsection{Determination of other steps and elements}

\subsubsection{Determination of input layer and output layer}

This paper selected some moments before the day t to predict randomly, which $\mathrm{X}_{1} \sim \mathrm{X}_{75}$ represent the actual loading value, $\mathrm{X}_{76} \sim \mathrm{X}_{93}$ represent the quantification value of the selected day's weather which includes maximum temperature, minimum temperature, average temperature, precipitation, and humidity, $\mathrm{X}_{94}$ represents day type data and the output layer represents the load value to be predicted.

\subsubsection{Data normalization process}

In order to increase the operating rate of neural network, data normalization process needs to be conductes. Similarity, we should to opposite data normalization process to the data of output layer. The specific formula can be seen as follows:

$$
L(d, t)^{\circ}=\frac{L(d, t)-L_{\min }}{L_{\max }-L_{\min }}
$$

Where $L(d, t)$ represents the input sample data before normalization process, $L_{\max }$ represents the maximum of the sample, $L_{\min }$ represents the maximum of the sample.

Moreover, the number of implication layer need to be satisfied as follows:

$$
\mathrm{H}=\sqrt{\mathrm{M}+\mathrm{N}}+\alpha
$$

Where $\mathrm{H}$ represents the the number of implication layer, $\mathrm{N}$ represents the node point number of input layer, $\mathrm{M}$ represents the Output layer node number. Employing the cut-and-trial can we get the the number of implication layer is 11.

\subsubsection{The selection of transmission function}

In the process of power load forecasting, the transmission function in the middle of the network layer is sigmoid function.

$$
f(x)=\frac{1}{1+e^{-x}}
$$

The transfer function in output layer adopts linear function purelin.

\subsection{Calculation of the model}

This paper adopted the data form December 1st to 30th of year 2015 as training sample to train the weight and threshold value of neural network and the same time adopted the data of December 31th as evaluating sample to evaluate the performance of neural network. Each input sample corresponding to the point-in-time is 94, one day's training sample is 96 groups (a time quantum is 15 minutes). By using MATLAB, adopting batch mode to train, and using BP neural network algorithm to train the training sample set, we can get the mean square error of curve as Fig. 1. And the one day's prediction effect is shown as Fig. 2.

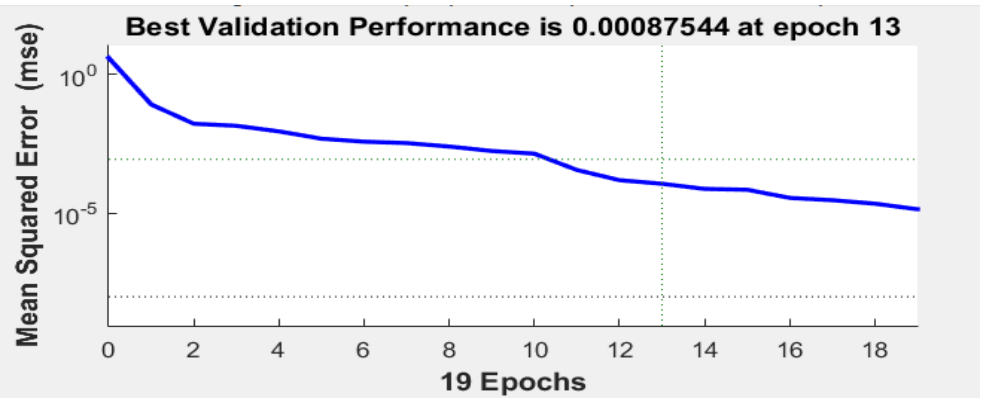

Fig. 1 the mean square error of curve 


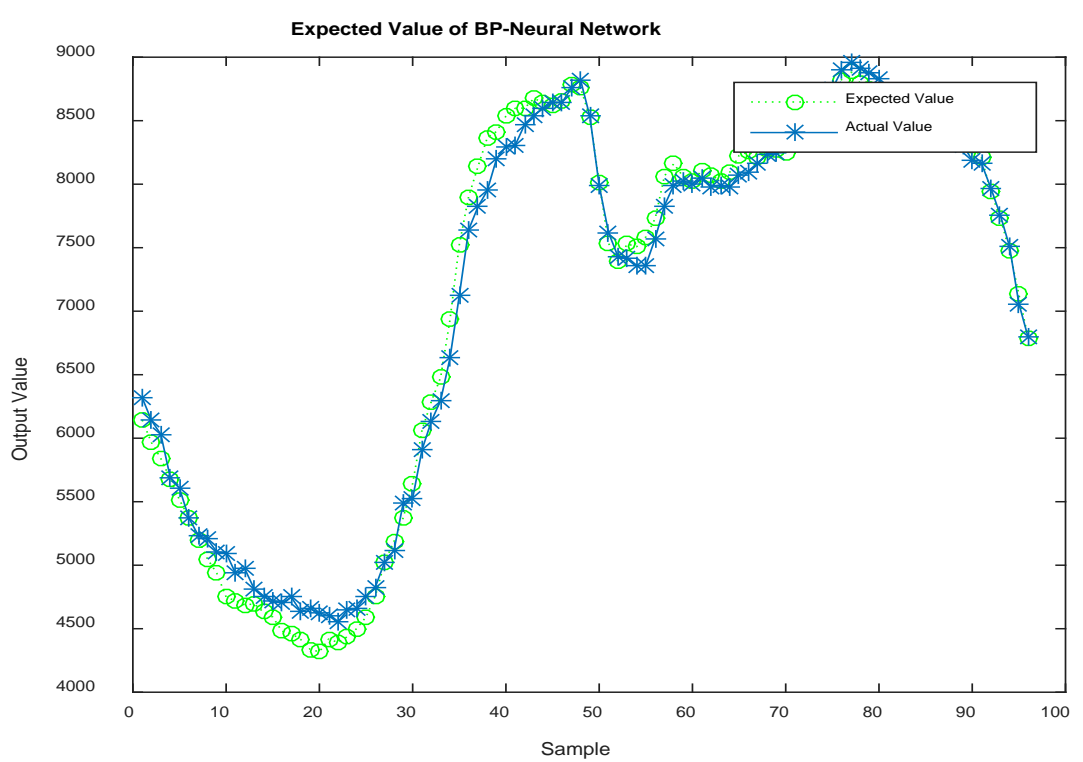

Fig.2 The actual curve and the predicted curve of Dec. 31th 2015

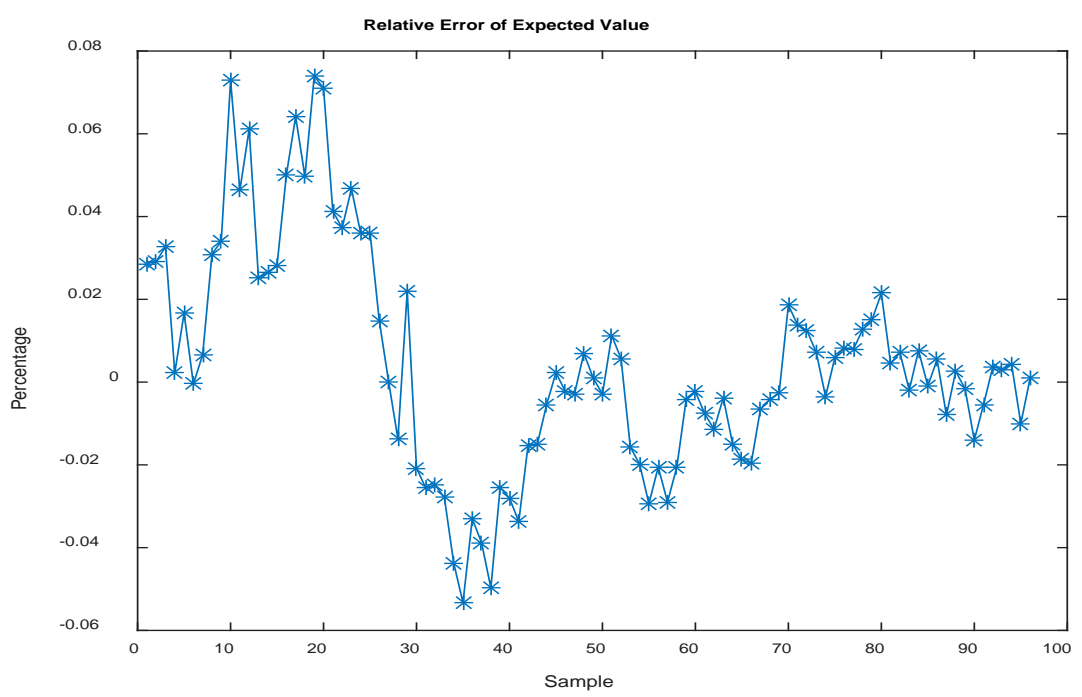

Fig.3 The relevant error curve of actual load and the predicted load of Dec. 31th 2015

From the Fig. 2 can we find that the predicted values fit in well with the actual value. From the Fig.3 can we find that the maximun relevence error between the predicted value and the actual value is approximately to $7 \%$, whereas the most relevence errors are under $4 \%$, the absolute error is less than 350, the error of mean square is approximately to 0.000014 . Then, we can get the conclusion that the load value predicted by BP nuetral network model is accurate.

\section{The comparison between the model considering weather and without considering weather}

According to the results of simulation by MATLAB, we can get the two model's training results as Fig. 4 and 5. And the concrete values can be seen as Tab. 3. 


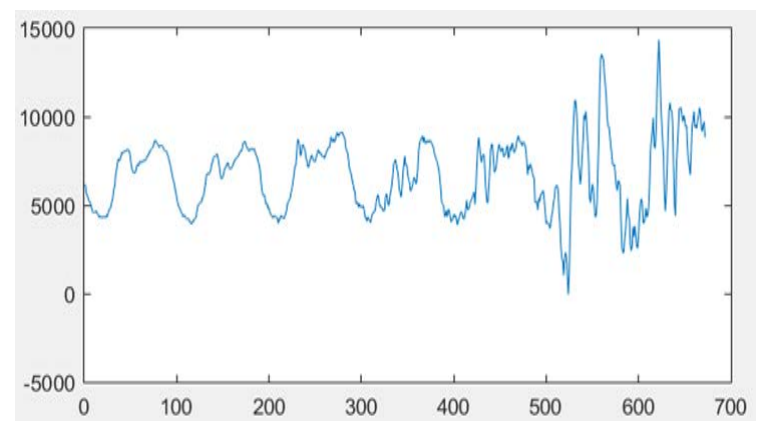

Fig.4 Week of load forcasting curve (without considering weather)

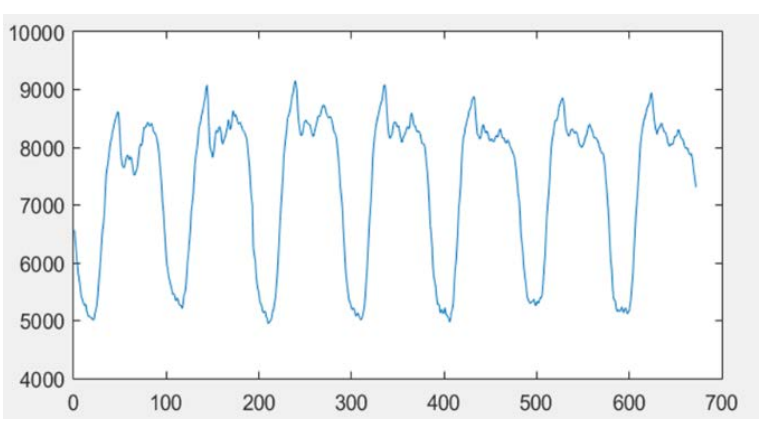

Fig. 5 Week of load forcasting curve (considering weather)

Tab. 3 The comparasion table of two models

\begin{tabular}{ccccc}
\hline model & $\begin{array}{c}\text { Input } \\
\text { value }\end{array}$ & $\begin{array}{c}\text { Iteration } \\
\text { time/second }\end{array}$ & $\begin{array}{c}\text { The sum of } \\
\text { absolute error }\end{array}$ & Error of mean squar \\
\hline Consider weather & 76 & 5 & 26000 & 0.0022 \\
$\begin{array}{c}\text { Without } \\
\text { considering } \\
\text { weather }\end{array}$ & 94 & 5 & 7900 & 0.0000141 \\
\hline
\end{tabular}

From the values in the error analysis table, we can find that the predicted value of the model considering weather is more accurate, weather in absolute error or the error of mean squar. At the same tiime, the prediction curve of the model considering weather embodys a strong regularity, whereas the model without considering weather is also accurate at the beginning but becomes disorder is the third day, which is go against the accurancy of the prediction.

\section{Conclusions}

This paper forecast the power load by using BP neural network model, taking the impact of weather factors into account, and found that the error is desirable, in addition, the neural network model considering meteorological factors is more stable and accurate, which has a positive effect on the load forecasting of power system.

\section{References}

[1] Zhou Chao,Wen-Yang Xing,Yu-Long Li. Review of power system load forecasting methods [J]. Journal of Power Supply. 2012,(6):32-39.

[2] Xiao-Ling Shen.Research on Power System Short-term Load Forecasting Approaches [D]. Tianjin University,2009.

[3] Gui-Qin Fu,Cao Xin,Qing-Ge Shi.Short-term power load forecast model based on the meteorological condition [C]. Annual meeting of Chinese Meteorological Society,2009. 